\title{
The Effect of Inhibitors on the Formation of Phosphoenolpyruvate by Rat Liver Mitochondria
}

\author{
BY W. BARTLEY AND B. DEAN \\ Department of Biochemistry, University of Sheffield, Sheffield 10
}

(Received 17 June 1969)

\begin{abstract}
1. Rat liver mitochondria oxidizing malate produce PEP (phosphoenolpyruvate) without the addition of ATP or other nucleotides. 2. The addition of oligomycin in the presence of 2,4-dinitrophenol did not abolish PEP formation and in some instances stimulated its formation. 3. Formation of PEP was inhibited by arsenate. 4. Arsenite decreased PEP formation and caused accumulation of pyruvate. 5. Added GTP and ITP had no effect on PEP formation. 6. PEP formed from malate in the presence of GTP and labelled $P_{1}$ had a specific radioactivity approximately the same as the $P_{i}$ with no contribution from the phosphate of the added GTP. 7. There was no parallelism between the effects of inhibitors on PEP formation from malate and their effects on the assayed activity of PEP carboxykinase. 8. In a direct comparison it was shown that the PEP carboxykinase content of mitochondria was insufficient to account for the PEP formation from malate. 9. Consideration of the kinetic characteristics of PEP carboxykinase and mitochondrial content of oxaloacetate and GTP show that this enzyme cannot account for the PEP formed from malate by mitochondria.
\end{abstract}

It is generally believed that the formation of PEP* from $\mathrm{C}_{4}$ dicarboxylic acids in mammals is brought about by the agency of PEP carboxykinase (EC 4.1.1.32; Utter \& Kurahashi, 1954), which carries out the reaction:

$$
\text { Oxaloacetate }+\mathrm{GTP} \rightleftharpoons \mathrm{PEP}+\mathrm{CO}_{2}+\mathrm{GDP}
$$

This enzyme is located partly in the mitochondria and partly in the cytosol, the proportions depending on the species. The amount of PEP carboxykinase has been shown to vary with the dietary state of the animal (Shrago, Young \& Lardy, 1964) and to be affected by hormones (Shrago, Lardy, Nordlie \& Foster, 1963). However, it is difficult to attribute PEP formation to the action of PEP carboxykinase in all experimental conditions. For example, Bartley \& Avi-Dor (1955) showed that PEP formation from malate was not inhibited by reagents that bind ketones. This suggests that free oxaloacetate could not be an intermediate in its formation. They also showed that extensive washing of tissue particles, which lowered the content of nucleotides to extremely low values, did not decrease the production of PEP and that in the presence of labelled $P_{1}$ the specific radioactivity of the $P E P$ was always above that of the terminal phosphate group of added nucleotides. Mudge, Neuberg \& Stanbury (1954) showed that PEP could still be produced from

* Abbreviation: PEP, phosphoenolpyruvate.
$\mathrm{C}_{4}$ dicarboxylic acids in the presence of 2,4-dinitrophenol and attributed this to a substrate phosphorylation accompanying the oxidative de. carboxylation of $\alpha$-oxoglutarate. The formation of PEP from $\mathrm{C}_{4}$ dicarboxylic acids may be stimulated by dinitrophenol (Gamble \& Mazur, 1967) and other uncoupling reagents (Davis \& Gibson, 1967) under circumstances where it is difficult to be sure that $\alpha$-oxoglutarate oxidation and substrate-level phosphorylation can supply the high-energy phosphate required. These experiments suggest the possibility of the formation of PEP by a route not involving PEP carboxykinase. Indirect support is given to this idea by the observation by Ballard \& Oliver (1963) that the production of PEP by rat liver mitochondria increased with age, whereas the content of mitochondrial PEP carboxykinase decreased with age (Ballard \& Hanson, 1967). The work described below gives further support to the idea that PEP formation by rat liver mitochondria does not involve PEP carboxykinase alone.

\section{MATERIALS}

Enzymes. Lactate dehydrogenase (E.C.1.1.1.27), malate dehydrogenase (E.C.1.1.1.37), glucose 6-phosphate dehydrogenase (E.C.1.1.1.49) and pyruvate kinase (E.C. 2.7.1.40) were crystalline suspensions in ammonium sulphate solution obtained from Boehringer Corp. (London) Ltd., London W.5. Hexokinase (E.C. 2.7.1.1) was Type III 
freeze-dried powder (12-16 units/mg.) from Sigma (London) Chemical Co. Ltd., London S.W.6.

Reagents. NAD, NADP, CoA and $3^{\prime} 5^{\prime}$-(cyclic)-AMP were from Boehringer Corp. (London) Ltd.; oligomycin, GSSG, GSH, DL-carnitine and ITP were from Sigma (London); 2,4-dinitrophenol was obtained from Hopkin and Williams Ltd., Chadwell Heath, Essex; quinolinic acid was from Koch-Light Laboratories Ltd., Colnbrook, Bucks.; D(t)biotin was from British Drug Houses Ltd., Poole, Dorset. GTP was from Calbiochem Ltd., London W.1.; sodium L-thyroxine was from Glaxo Laboratories Ltd., Greenford, Middlesex; dicoumarol was from Organon Laboratories Ltd., London W.C.2; sodium amytal was from Eli Lilly and Co. Ltd., Basingstoke, Hants.; triethyltin sulphate was a gift from Dr W. N. Aldridge, Medical Research Council Toxicology Research Unit, Woodmansterne Road, Carshalton, Surrey; L-phosphomalic acid was prepared by Dr D. H. Williamson; fluoromalate was prepared by Dr P. L. K. Kent; vitamin $B_{12}$ coenzyme was a gift from $\mathrm{Dr}$ J. R. Guest, Department of Microbiology, University of Sheffield; acetyl-CoA was prepared from $\mathrm{CoA}$ and acetic anhydride as described by Stadtman (1957); $\mathrm{KH}_{2}{ }^{32} \mathrm{PO}_{4}$ (carrier-free) was obtained from The Radiochemical Centre, Amersham, Bucks. Other reagents were the purest commercially available.

Rats. These were adult males of a Wistar strain, weighing between 175 and $250 \mathrm{~g}$.

\section{METHODS}

Preparation of mitochondria. Rats were killed by stunning and decapitation and livers were removed at once into $0.25 \mathrm{M}$-sucrose in 5mM-tris-HCl buffer, $\mathrm{pH7.4}$, at $0^{\circ}$. Mitochondria were prepared essentially as described by Werkheiser \& Bartley (1957) except that the final wash and suspension was done in the sucrose medium after dissecting the mitochondrial pellet away from contaminating erythrocytes with a stainless-steel spatula.

The inclusion of nicotinamide, EDTA and 2-mercaptoethanol in the preparation medium had no stimulatory effect on PEP production and these additives were not therefore used. Carrying out the final rinse and resuspension in medium containing $\mathrm{KCl}$ decreased the activity of the soluble enzyme lactate dehydrogenase from $1.5 \%$ to $0.5 \%$ of its activity in the whole homogenate and caused about a 30\% decrease in PEP yield and about $10 \%$ decrease in $\mathrm{O}_{2}$ uptake.

State of preservation of the mitochondria. Suspension of rat liver mitochondria (prepared in $0.25 \mathrm{M}$-sucrose) in salt solutions causes mitochondrial swelling, a loss of mitochondrial protein and nucleotides and greatly increased permeability to solutes (Bartley \& Amoore, 1958; Amoore \& Bartley, 1958; Bartley, 1961; Bartley \& Enser, 1964) and loss of internal amino acids (Alberti \& Bartley, 1969). Such changes are accelerated when the mitochondria are suspended in solutions of low ionic strength. Swelling agents such as dinitrophenol also disrupt membrane structure (for review of mitochondrial swelling see Lehninger, 1962). Thus the mitochondrial preparation used would be expected to show minimal permeability restrictions to the passage of solutes. The electron microscope picture of the material used (Plate 1) is in accordance with this assumption. However it is impossible to prove that restrictions to penetration of some solutes to some areas of the disrupted mitochondria do not remain and the experiments presented below must be viewed in this light.

Incubations were carried out in a Warburg apparatus containing the following reagents: $0.9 \% \mathrm{KCl}$ in $10 \mathrm{~mm}$ -

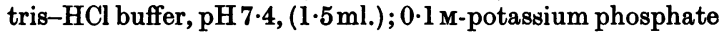
buffer, pH 7.4, (0.05 ml.); 0.1 M-potassium malate $(0.1 \mathrm{ml}$.); other additions as required and water to $3.5 \mathrm{ml}$. were placed in the main compartment. The mitochondrial suspension $(0.5 \mathrm{ml}$. of $200 \mathrm{mg}$. wet wt. $/ \mathrm{ml}$. $)$ in the sucrose-tris mixture was placed in the side arm to give $20-25 \mathrm{mg}$. dry wt. of mitochondria/vessel. The centre well contained $0.2 \mathrm{ml}$. of $2 \mathrm{M}-\mathrm{NaOH}$ and filter paper, to absorb $\mathrm{CO}_{2}$. Vessels were attached to the manometers and shaken in the bath at $25^{\circ}$ for $5 \mathrm{~min}$. before tipping. Incubation was usually for $20 \mathrm{~min}$., after which the vessels were removed on to ice and the reaction was stopped by addition of $0.8 \mathrm{ml}$. of $3 \mathrm{M}-\mathrm{HClO}_{4}$. The gas phase was usually air; oxygen when used had no effect on respiration rate or PEP formation. After removal of proteins by centrifugation the vessel contents were partially neutralized with $0 \cdot 18 \mathrm{ml}$. of $60 \%(\mathrm{w} / \mathrm{v}) \mathrm{KOH}$ and final adjustment to $\mathrm{pH} 7 \cdot 0$ was made by dropwise addition of $2 \mathrm{M}-\mathrm{KHCO}_{3}$. After cooling in ice for $5 \mathrm{~min}$. precipitated $\mathrm{KClO}_{4}$ was spun down and the supernatant diluted to $5 \mathrm{ml}$.

Analytical methods. PEP and pyruvate were determined together by the spectrophotometric method of Czok \& Eckert (1963) with lactate dehydrogenase and pyruvate kinase, the only modification being that potassium phosphate buffer was substituted for triethanolamine buffer.

PEP carboxykinase was assayed either as described by Ballard \& Hanson (1967) in the direction of oxaloacetate formation or as described by Seubert \& Huth (1965) and Yeung \& Oliver (1967) in the other direction.

ATP was determined spectrophotometrically with hexokinase and glucose 6-phosphate dehydrogenase as described by Lamprecht \& Trautschold (1963). When hexokinase and glucose were added to the Warburg vessels during incubation the glucose 6-phosphate formed was measured by using the second stage of this assay.

\section{RESULTS}

Minimum system for the formation of PEP. As Bartley (1955) and Gamble \& Mazur (1967) have shown, added ATP is unnecessary for the formation of PEP in pigeon liver or rabbit liver mitochondria. Similarly in the rat liver mitochondrial preparations used in this work there was no dependence on exogenous ATP for PEP formation, as may also be deduced from Gamble \& Mazur (1967). In the work described below ATP was omitted, as was magnesium, which also failed to stimulate PEP formation. Malate was the usual substrate, and Fig. 1 shows the effect of substrate concentration on PEP production, oxygen uptake and on pyruvate formation. At 1 mM-malate the rate of PEP formation and the rate of oxygen uptake had reached a maximum but pyruvate production continued to increase rapidly up to $2.5 \mathrm{~mm}$-malate and thereafter increased slowly without reaching a maximum.

At saturating concentrations of malate (above 


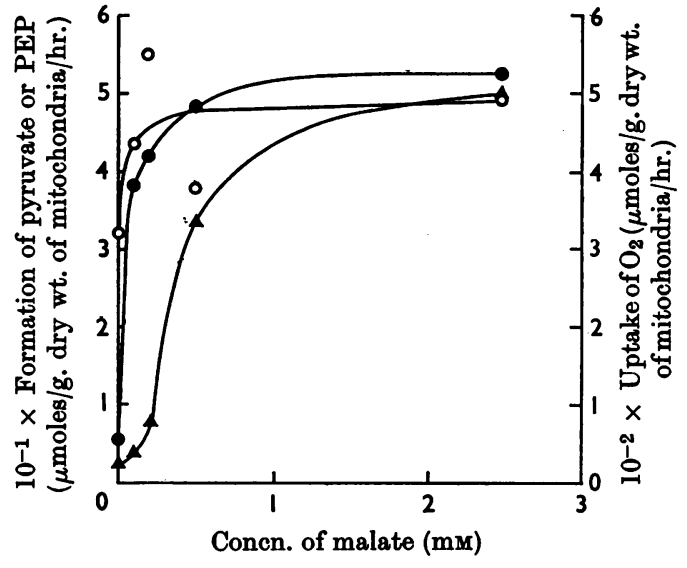

Fig. 1. Effect of malate concentration on oxygen uptake and PEP and pyruvate formation. O, Oxygen; $\bullet$, PEP; $\Delta$, pyruvate.

$5 \mathrm{~mm})$, PEP formation increased with phosphate concentrations up to $1.25 \mathrm{mM}$, but higher concentrations of phosphate had no further effect. In most experiments, therefore, the malate concentration was $2.5 \mathrm{~mm}$ and the phosphate concentration was $1.25 \mathrm{~mm}$. Any different conditions are indicated in the legends to the figures or tables. PEP production was not affected by the osmolarity of the suspension medium between 120 and $250 \mathrm{~m}$-osmolal. Routinely $120 \mathrm{~m}$-osmolal was used, in which the mitochondria were swollen and partially disrupted, as shown by electron microscopy (Plate 1).

Effect of 2,4-dinitrophenol on PEP and pyruvate formation from malate. Stanbury \& Mudge (1954) showed that PEP formation from $\alpha$-oxoglutarate was stimulated by dinitrophenol. Gamble \& Mazur (1967) showed in the rabbit liver that variation of the dinitrophenol concentration produced a biphasic response, which was partially dependent on the time of incubation. With rat liver mitochondria a similar biphasic response was found with peaks at about $0.01 \mathrm{~mm}$ and $0.1 \mathrm{~mm}$, but the concentration of dinitrophenol required varied somewhat depending on the mitochondrial preparation. However, $0.05 \mathrm{~mm}$-dinitrophenol always produced an inhibition of PEP formation On average, the stimulation by $0.01 \mathrm{~mm}$-dinitrophenol was $25 \%$ and, on average, 0.1 mM-dinitrophenol inhibited by $7 \%$, but the mean inhibition with $0.05 \mathrm{~mm}$-dinitrophenol was $75 \cdot 8 \%$. In the absence of dinitrophenol the mean PEP production was $56.5 \mu \mathrm{moles} / \mathrm{g}$. dry wt. of mitochondria/hr.

Although dinitrophenol often stimulated PEP formation from malate, it always inhibited pyruvate formation. In the absence of dinitrophenol, the mitochondria produced $22 \cdot 4 \mu \mathrm{moles}$ of pyruvate/g. dry wt./hr.; at 0.01 mM-dinitrophenol, production of pyruvate was only $23 \%$ of this and, at $0.1 \mathrm{~mm}, 14 \%$. The oxygen uptake was stimulated by dinitrophenol at $0.01 \mathrm{~mm}$ by $85 \%$, at $0.05 \mathrm{~mm}$ by $22 \%$ and at $0.1 \mathrm{~mm}$ by $61 \%$. Thus, at all concentrations of dinitrophenol in this range, there was evidence of uncoupling, as judged by the stimulation of oxygen uptake. In the absence of dinitrophenol, about $6 \mu$ moles of oxygen were consumed/ $\mu$ mole of PEP produced and in the presence of dinitrophenol about $10 \mu \mathrm{moles}$ were consumed $/ \mu \mathrm{mole}$ of PEP produced.

Effect of dinitrophenol on the concentration of ATP found in the mitochondria. Although there are many experiments showing that dinitrophenol lowers the phosphorylation quotient, there appear to be no measurements of concentrations of mitochondrial ATP under our experimental conditions. Table 1 shows the change with time of the ATP and PEP concentrations when mitochondria are incubated with malate alone or with dinitrophenol at three different concentrations. Even in the presence of 0.1 mM-dinitrophenol, ATP was found after incubation. Though the amount fell progressively as the concentration of dinitrophenol increased, the fall was not accompanied by a fall in PEP formation. Thus, comparison of the amounts of high-energy phosphate present after incubation for $30 \mathrm{~min}$. show that, the PEP concentration in one case doubled when the ATP concentration halved (value with 0.01 mM-dinitrophenol compared with value without dinitrophenol) and in another case the PEP concentration increased by $48 \%$ when the ATP concentration diminished by $\mathbf{7 7 \%}$ (value with 0.1 mM-dinitrophenol compared with value without dinitrophenol). In an effort to decrease the concentration of ATP in the system, yeast hexokinase and glucose were added to the incubation medium and the glucose 6-phosphate formed was measured as well as the PEP (Table 2). Almost twice as much PEP was formed when hexokinase and glucose were added and, in the absence of dinitrophenol, almost twice as much glucose was phosphorylated as there was PEP formed. Since rat liver mitochondria contain a high activity of nucleoside diphosphate kinase (Ishihara \& Kikuchi, 1968), any decrease in ATP would be expected to be accompanied by a corresponding decrease in GTP; under these conditions the activity of PEP carboxykinase would, therefore be expected to decrease progressively.

Effect of oligomycin on PEP formation. Oligomycin is an inhibitor of the transphosphorylation processes of oxidative phosphorylation and might therefore be expected to diminish PEP formation if ATP was the source of the high-energy phosphate thereof. Table 3 shows that respiration was diminished in the presence of oligomycin. This diminution in respiration is taken as a measure of the 
Table 1. Effect of the concentration of 2,4-dinitrophenol on the time-course of formation of PEP and on the endogenous concentration of ATP

Washed rat liver mitochondria were incubated in Warburg vessels at $25^{\circ}$ in the medium described in the Methods section with 2,4-dinitrophenol at the concentrations given below. Samples were removed immediately and at the times indicated, and deproteinized with ice-cold $3 \mathrm{M}-\mathrm{HClO}_{4}$ (final concn. $0 \cdot 5 \mathrm{M}$ ). After removal of protein the supernatants were neutralized and the contents of PEP and ATP determined as described in the Methods section. All values are given as $\mu$ moles of material found/g. dry wt. of mitochondria.

Incubation time (min.)

\begin{tabular}{|c|c|c|c|c|}
\hline \multirow[b]{2}{*}{ Final dinitrophenol concn. } & & & & \\
\hline & $\ldots$ & 0.01 & 0.05 & $0 \cdot 1$ \\
\hline 0 & $\mathbf{0}$ & $\mathbf{0}$ & $\mathbf{0}$ & $\mathbf{0}$ \\
\hline 2 & 0.94 & $4 \cdot 0$ & $0 \cdot 33$ & $1 \cdot 83$ \\
\hline 5 & $1 \cdot 36$ & $4 \cdot 41$ & $3 \cdot 06$ & $3 \cdot 85$ \\
\hline 30 & $11 \cdot 5$ & $26 \cdot 7$ & $10 \cdot 2$ & $16 \cdot 7$ \\
\hline
\end{tabular}

Concn. of PEP

$$
\text { (mM) }
$$

\begin{tabular}{llll}
\multicolumn{4}{c}{$\begin{array}{c}\text { Concn. of ATP } \\
(\mathrm{mM})\end{array}$} \\
\hline 0 & 0.01 & 0.05 & 0.1 \\
1.55 & 0.85 & 0.42 & 0.56 \\
1.41 & 1.45 & 1.41 & 1.17 \\
1.03 & 1.83 & 0.85 & 1.78 \\
4.84 & 2.40 & 1.88 & 1.13
\end{tabular}

Table 2. Effect of hexokinase and 2,4-dinitrophenol on the formation of PEP and the phosphorylation of glucose

Incubation was in Warburg vessels: final vol. $4 \mathrm{ml}$. and other conditions as described in Table 1, except that hexokinase (30 units) and glucose $(100 \mu$ moles) were present where indicated and the incubation time was for $15 \mathrm{~min}$. after tipping in the mitochondria. Values are expressed as $\mu$ moles of substance formed $/ g$. dry wt. $/ 30 \mathrm{~min}$. incubation.

$\begin{array}{cccc}\begin{array}{c}\text { Dinitrophenol } \\ \text { concn. } \\ (\mathrm{mM})\end{array} & \begin{array}{c}\text { Hexokinase } \\ \text { +glucose }\end{array} & \begin{array}{c}\text { PEP } \\ \text { formed }\end{array} & \begin{array}{c}\text { Glucose } \\ \text { 6-phosphate } \\ \text { formed }\end{array} \\ 0 & - & 4 \cdot 6 & - \\ 0 & + & 8 \cdot 6 & 15 \cdot 3 \\ 0.01 & + & 29 \cdot 2 & 7 \cdot 2 \\ 0.05 & + & 16 \cdot 3 & 0 \\ 0 \cdot 1 & + & 19 \cdot 1 & 0\end{array}$

degree of coupling of respiration with phosphorylation. The uncoupled respiration is still capable of causing the formation of PEP. Addition of dinitrophenol overcame the inhibition by oligomycin and allowed the stimulation of PEP production. These results are diametrically opposed in every detail to those of Gevers (1967) with pigeon liver mitochondria, in which PEP formation is known to be due to PEP carboxykinase, which is exclusively located in the particles.

Effect of triethyltin, arsenate, dicoumarol and thyroxine on PEP formation. These substances might all be expected to diminish the yield of PEP if ATP production is involved in its formation. Table 4 shows that arsenate at both $125 \mathrm{~mm}$ and $12.5 \mathrm{~mm}$ greatly decreased PEP production (approx. $90 \%$ ) and that further addition of dinitrophenol or oligomycin did not change the effect of arsenate. However, these results are also compatible with arsenate acting by arsenolysis of a primary high-
Table 3. Effect of oligomycin and 2,4-dinitrophenol on the production of PEP from malate

Experimental conditions were as described in Table 1. PEP formation and $\mathrm{O}_{2}$ uptake are expressed as $\mu$ moles/g. dry wt. of mitochondria.

\begin{tabular}{ccccc} 
Expt. no. & $\begin{array}{c}\text { Concn. of } \\
\text { oligomycin } \\
(\mu \mathrm{g} . / \mathrm{ml} .)\end{array}$ & $\begin{array}{c}\text { Concn. of } \\
\text { dinitro- } \\
\text { phenol } \\
(\mathrm{mM})\end{array}$ & $\begin{array}{c}\mathrm{O}_{2} \\
\text { uptake }\end{array}$ & $\begin{array}{c}\text { PEP } \\
\text { formed }\end{array}$ \\
1 & 0 & 0 & 147 & $23 \cdot 8$ \\
& 5 & 0 & 115 & $12 \cdot 9$ \\
& 0 & $0 \cdot 25$ & 296 & $27 \cdot 6$ \\
& 5 & $0 \cdot 25$ & 310 & $37 \cdot 0$ \\
& 0 & 0 & 170 & $27 \cdot 7$ \\
& 5 & 0 & 96 & $7 \cdot 4$ \\
& 0 & $0 \cdot 01$ & 333 & $43 \cdot 4$ \\
& 5 & $0 \cdot 01$ & 296 & $31 \cdot 2$ \\
& 20 & 0 & 91 & $8 \cdot 5$ \\
& 20 & $0 \cdot 01$ & 295 & $29 \cdot 6$ \\
& 0 & $0 \cdot 1$ & 294 & $22 \cdot 6$ \\
& 5 & $0 \cdot 1$ & 288 & $38 \cdot 8$ \\
& 20 & $0 \cdot 1$ & 264 & $40 \cdot 0$ \\
& & & & \\
\hline
\end{tabular}

energy phosphate intermediate arising either from oxidative phosphorylation or from substrate-level phosphorylation.

With triethyltin there was no effect on the formation of PEP nor on the associated oxygen uptake. The addition of DNP decreased the yield of PEP in the presence of triethyltin but the effect was diminished as the concentration of dinitrophenol was raised from $0.01 \mathrm{~mm}$ to $0.1 \mathrm{~mm}$. Respiration was increased only proportionally to the relief of inhibition of PEP formation by the increased concentration of dinitrophenol. Dicoumarol had little effect on PEP production of respiration. Thyroxine also had little influence.

The lack of effects of triethyltin, dicoumarol and 


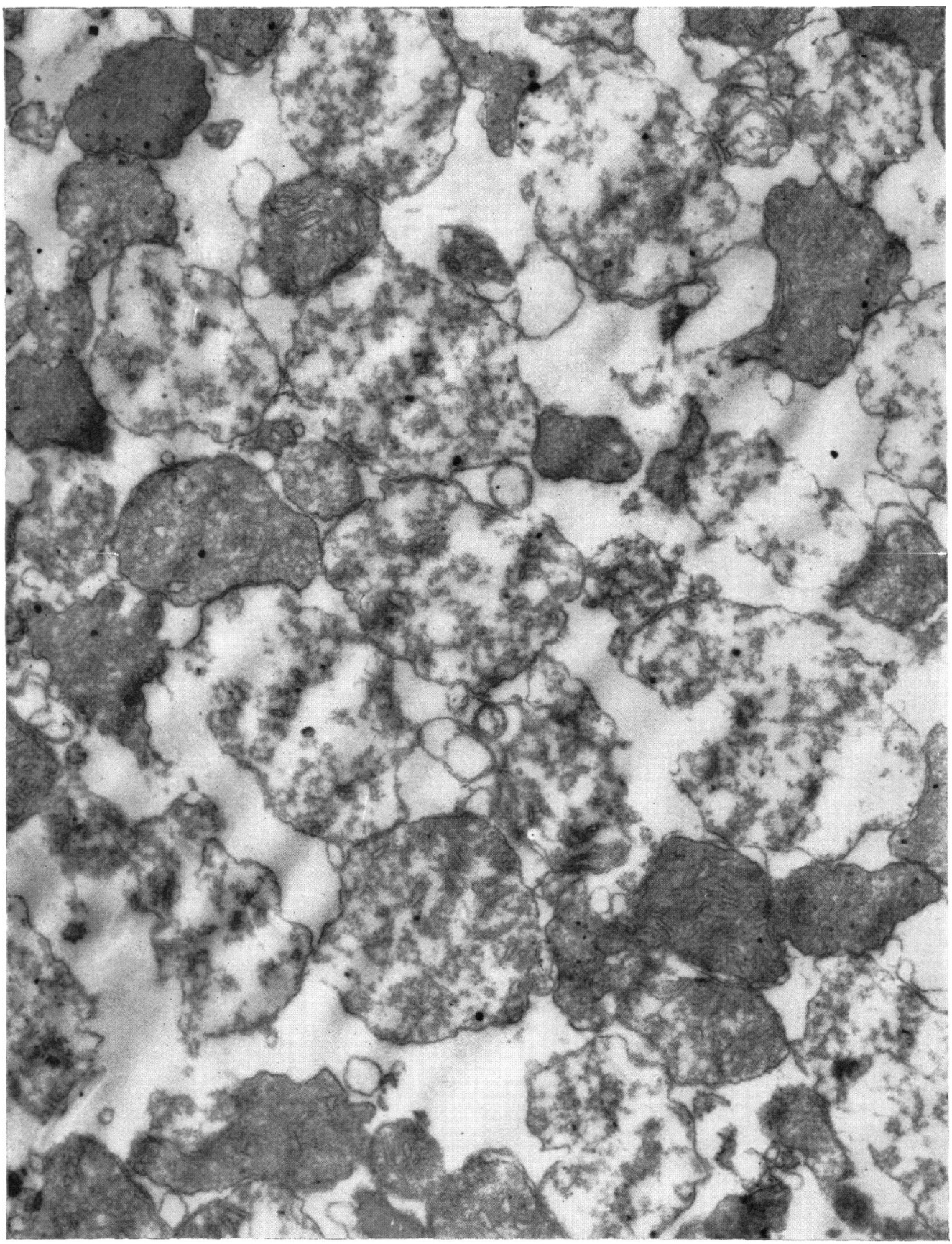

EXPLANATION OF PLATE I

Electron micrograph of mitochondria used in the experiments. Most of the mitochondria are swollen, with ruptured membranes. Magnification $\times 25000$. 
thyroxine all support the idea that PEP formation does not depend on the prior synthesis of ATP.

Effect of phosphomalate, fluoromalate, Amytal and quinolinate on PEP formation. Table 5 shows the effect of these inhibitors on PEP formation. The effect of the first three can be explained as due to an inhibition of malate oxidation. Quinolinate is an

Table 4. Effect of arsenate, triethyltin, dicoumarol and thyroxine on PEP formation

All results are given as $\mu$ moles of substance/g. dry wt. of mitochondria/hr. Other details are as described in Table 1.

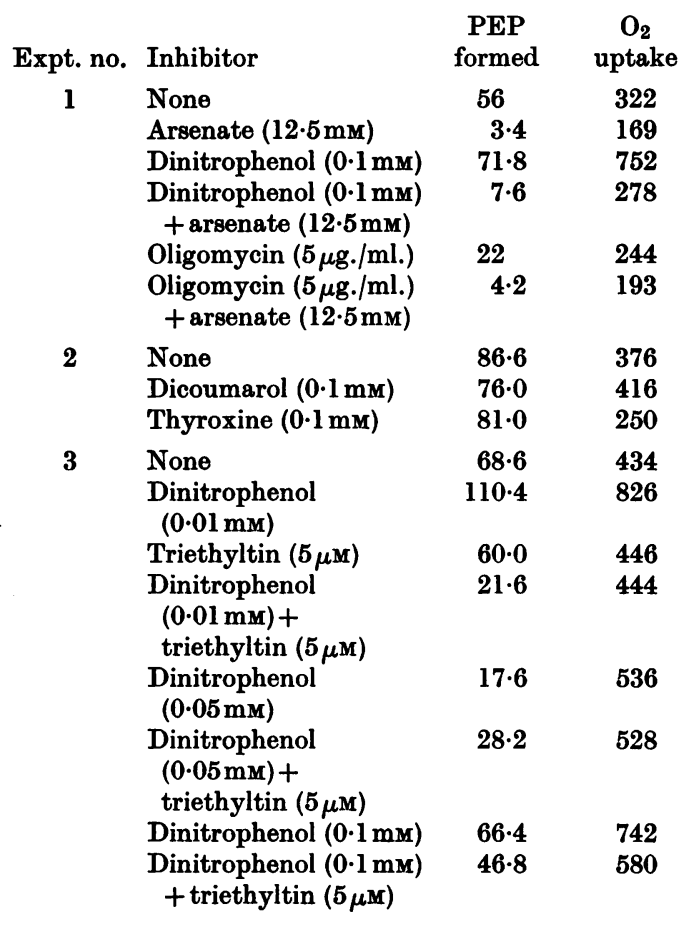

inhibitor of PEP carboxykinase (Veneziale, Walter, Kneer \& Lardy, 1967) and as shown below would be expected to inhibit that enzyme's activity by $60 \%$. If the $30 \%$ inhibition that was found is a measure of the contribution of PEP carboxykinase to the production of PEP in the absence of dinitrophenol the remainder must be formed by a route not involving PEP carboxykinase. This is confirmed by the observation that PEP formation in the presence of dinitrophenol and quinolinate was $92 \%$ of that in the presence of dinitrophenol alone or $114 \%$ of that in the absence of dinitrophenol. Any explanation of this finding on the basis of a formation of PEP by PEP carboxykinase would require a stimulation of the enzyme activity by dinitrophenol and, as shown below, this does not occur.

Effect of arsenite on PEP and pyruvate production. Gamble \& Mazur (1967) in their study of PEP formation in rabbit liver mitochondria showed a differential effect of arsenite (which blocks $\alpha$ oxoglutarate oxidation and thus inhibits substratelevel phosphorylation of GDP) on PEP production depending on whether malate or $\alpha$-oxoglutarate was the substrate. Whereas $0 \cdot 1 \mathrm{~mm}$-arsenite caused a $50 \%$ inhibition of PEP production from $\alpha$-oxoglutarate there was virtually no effect when malate was the substrate. From the lack of inhibition with arsenicals, Gamble \& Mazur (1967) deduced that GTP was not concerned in the production of PEP when malate was the substrate.

Rat liver mitochondria appear to behave differently from rabbit liver mitochondria in response to arsenite. As shown in Table 6 arsenite very greatly decreased PEP production but greatly stimulated pyruvate production. These results suggest the involvement of a dithiol group in the formation of PEP. If this is so, a similar effect should be obtained with $\mathrm{Cd}^{2+}$ (Sanadi, Langley \& White, 1959). Experiments with $0.01 \mathrm{~mm}-\mathrm{Cd}^{2+}$ showed that PEP production was inhibited and pyruvate formation stimulated to the extent found in the presence of

Table 5. Effect of phosphomalate, fluoromalate, Amytal and quinolinate on PEP formation

The amount of PEP produced in absence of inhibitors is given as $100 \%$.

$\begin{array}{lcccc}\text { Inhibitor } & \begin{array}{c}\text { Concn. } \\ (\mathrm{mM})\end{array} & \begin{array}{c}\text { PEP formed } \\ (\% \text { of control) }\end{array} & \begin{array}{c}\text { when added } \\ (\mathrm{mM})\end{array} & \begin{array}{c}\text { presence of dinitro- } \\ \text { phenol) }\end{array} \\ \text { Phosphomalate } & 1 & 60 & 0 \cdot 01 & 82 \\ \text { Fluoromalate } & 12 \cdot 5 & 5 \cdot 6 & 0 \cdot 01 & 0 \cdot 2 \\ \text { Amytal } & 0 \cdot 25 & 82 & 0 \cdot 01 & -2 \\ \text { Quinolinate } & 2 & 16 \cdot 3 & - & - \\ \end{array}$

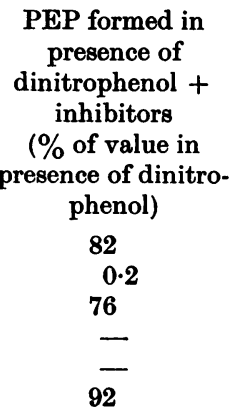


Table 6. Effect of arsenite on the formation of PEP and pyruvate from malate

All results are expressed as $\mu$ moles of substance/g. dry wt. of mitochondria/hr. Other details are as given in Table 1 or the Methods section.

\begin{tabular}{|c|c|c|c|c|c|}
\hline Expt. no. & $\begin{array}{l}\text { Concn. of arsenite } \\
\text { (mм) }\end{array}$ & $\begin{array}{l}\text { Concn. of } \\
\text { dinitrophenol } \\
\text { (mM) }\end{array}$ & PEP formed & Pyruvate formed & $\mathrm{O}_{2}$ uptake \\
\hline \multirow[t]{8}{*}{1} & $\mathbf{0}$ & $\mathbf{0}$ & $58 \cdot 8$ & $20 \cdot 4$ & 402 \\
\hline & 2 & 0 & $4 \cdot 2$ & $92 \cdot 8$ & 153 \\
\hline & $\mathbf{0}$ & 0.01 & $60 \cdot 0$ & $4 \cdot 4$ & 730 \\
\hline & 2 & 0.01 & $4 \cdot 8$ & $91 \cdot 8$ & 166 \\
\hline & 0 & 0.05 & $21 \cdot 0$ & $2 \cdot 6$ & 520 \\
\hline & 2 & 0.05 & $4 \cdot 8$ & $79 \cdot 2$ & 181 \\
\hline & 0 & $0 \cdot 1$ & $41 \cdot 2$ & 0 & 598 \\
\hline & 2 & $0 \cdot 1$ & $5 \cdot 8$ & $78 \cdot 8$ & 208 \\
\hline \multirow[t]{8}{*}{2} & 0 & 0 & $60 \cdot 6$ & $8 \cdot 8$ & 516 \\
\hline & $0 \cdot 1$ & $\mathbf{0}$ & $17 \cdot 6$ & $62 \cdot 0$ & 316 \\
\hline & 0 & 0.01 & $51 \cdot 6$ & $2 \cdot 4$ & 750 \\
\hline & $0 \cdot 1$ & 0.01 & $6 \cdot 8$ & $24 \cdot 6$ & 368 \\
\hline & 0 & 0.05 & $10 \cdot 1$ & $1 \cdot 0$ & 476 \\
\hline & $0 \cdot 1$ & 0.05 & $6 \cdot 4$ & $10 \cdot 4$ & 402 \\
\hline & 0 & $0 \cdot 1$ & $14 \cdot 2$ & 1.6 & 484 \\
\hline & $0 \cdot 1$ & $0 \cdot 1$ & $10 \cdot 8$ & $3 \cdot 8$ & 430 \\
\hline
\end{tabular}

arsenite. The addition of $0.01 \mathrm{~mm}$-dinitrophenol did not alter this effect.

Two other experimental conditions caused an increase in pyruvate production. If all potassium salts were replaced by sodium, there was a $20 \%$ diminution in PEP formation but a fivefold increase in pyruvate production. In the presence of $10 \mathrm{~mm}$-fluoride there was a fivefold stimulation in pyruvate production and a $35 \%$ inhibition of PEP formation. However, the addition of dinitrophenol completely abolished the pyruvate formation and, except at the higher concentrations of dinitrophenol, there was a lowered production of PEP when fluoride was present. The percentage yields of PEP in the presence of fluoride compared with those in its absence were: with $0.01 \mathrm{~mm}$-dinitrophenol, $58 \%$; with $0.05 \mathrm{~mm}$-dinitrophenol, $135 \%$; and with $0.1 \mathrm{~mm}$ dinitrophenol, $146 \%$. The effect of added disulphide or thiol was tested by adding GSSG or GSH. GSSG (2.5 mM) gave a $50 \%$ inhibition of PEP production in the absence of dinitrophenol but had no effect on the stimulated PEP production brought about by $0.25 \mathrm{~mm}$-dinitrophenol. GSH $(10 \mathrm{~mm})$ inhibited the dinitrophenol-stimulated PEP production by $90 \%$ and GSSG (5mM) inhibited it by $40 \%$. In no case was there a marked effect on pyruvate formation.

Effect of $\mathrm{Ca}^{2+}$ on PEP formation. Since $\mathrm{Ca}^{2+}$ is known to uncouple oxidative phosphorylation, it might be expected that there would be a stimulation of PEP production as with dinitrophenol. However, this was not the case; in the presence of 0.25 mM-dinitrophenol, $0.125 \mathrm{~mm}-\mathrm{Ca}^{2+}$ inhibited PEP production by $35 \%$ and decreased it to the value obtained in the absence of dinitrophenol; at
$0.5 \mathrm{mM} \cdot \mathrm{Ca}^{2+}$, the inhibition was $58 \%$, at $2 \mathrm{mM}, 92 \%$ and at $5 \mathrm{~mm}, 96 \%$. At this concentration of $\mathrm{Ca}^{2+}$ the respiration was only $20 \%$ of the rate in the absence of the cation.

Effect of possible cofactors. The following substances had little or no effect on PEP production: NAD, NADP, CoA, biotin, vitamin $B_{12}$ coenzyme, $3^{\prime}, 5^{\prime}$ (cyclic)-AMP, carnitine; in one experiment acetylCoA $(0.25 \mathrm{~mm})$ gave a $60 \%$ inhibition of PEP production. It is specially noteworthy that GTP and ITP did not stimulate PEP production.

Incorporation of labelled $P_{i}$ into PEP. A series of experiments was carried out in which mitochondria prepared and washed in $0.25 \mathrm{M}$-sucrose were (1) suspended in $0.12 \mathrm{M}$-potassium chloride-0.035 M imidazole buffer, $\mathrm{pH} 7 \cdot 6$; (2) suspended in $0.035 \mathrm{M}$-imidazole buffer, $\mathrm{pH} 7.6$; or (3) suspended in water at $0^{\circ}$ for $15 \mathrm{~min}$., centrifuged down and resuspended in water with $0.035 \mathrm{M}$-imidazole buffer, pH 7.6. In all cases the treated mitochondria were then added to the standard mixture of potassium chloride, dinitrophenol and water in Warburg vessels. The side arms of the vessels contained malate as substrate, 4 or $8 \mu$ moles of GTP and labelled $P_{1}$. After equilibration of the vessels the contents of the side arms were tipped and the respiration was measured with time. Vessels were removed at intervals, the reactions stopped and the specific radioactivity of the phosphate compounds determined after separation by paper chromatography or t.l.c. with toluene-p-sulphonic acid2-methylbutan-2-ol-water (Hanes \& Isherwood, 1949). At all times the specific radioactivity of the $P E P$ reflected that of the $P_{1}$ and at the longer times 
even rose above that of the $P_{1}$. If the $P E P$ formation had involved PEP carboxykinase no radioactivity would be expected in the PEP since the added GTP was unlabelled. The possibility that there is a small pool of endogenous GTP that becomes rapidly labelled and which does not equilibrate with the added GTP appears unlikely because of the disruptive procedures used in preparing the mitochondria.

Effect of inhibitors on PEP carboxykinase. The various inhibitors that were shown to affect PEP production were used in the same concentrations in the assay system for PEP carboxykinase described by Ballard \& Hanson (1967). This techniquemeasures the incorporation of ${ }^{14} \mathrm{CO}_{2}$ into oxaloacetate when PEP is added to the system. Table 7 shows the results; in no case was there a parallelism between the effect of the inhibitors on PEP carboxykinase and the effect on the formation of PEP in the rat liver mitochondrial system. In particular it should be noted that the biphasic response to dinitrophenol given by the mitochondria was not shown in the enzyme assay.

Comparison of PEP production by PEP carboxykinase and by mitochondria. A preparation of mitochondria (200 mg. wet wt. $/ \mathrm{ml}$.) was divided into two parts. One part was incubated with malate and DNP under the standard conditions already described and the other part was subjected to ultrasonic disintegration ( $2 \times 1 \mathrm{~min}$. in MSE disintegrator with full power while cooling in ice) and spun at $33000 \mathrm{~g}$ for $10 \mathrm{~min}$. to obtain a clear supernatant.

Table 7. Effect of inhibitors and uncouplers of PEP carboxykinase assay compared with their effect on $P E P$ production by incubated mitochondria

Results are expressed as $100 \times$ (inhibited value/noninhibited value).

\begin{tabular}{|c|c|c|}
\hline Inhibitor or uncoupler & $\begin{array}{c}\text { carboxykinase } \\
\text { assay }\end{array}$ & $\begin{array}{l}\text { by incubated } \\
\text { mitochondria }\end{array}$ \\
\hline Dinitrophenol $0.01 \mathrm{~mm}$ & 96 & 125 \\
\hline Dinitrophenol $0.05 \mathrm{~mm}$ & $95 \cdot 5$ & $24 \cdot 2$ \\
\hline Dinitrophenol $0.1 \mathrm{~mm}$ & $103 \cdot 5$ & 93 \\
\hline Arsenite (2mM) & 106 & $7 \cdot 15$ \\
\hline Arsenate $(12.5 \mathrm{mM})$ & 88 & $6 \cdot 1$ \\
\hline Triethyltin $(0 \cdot 1 \mathrm{~mm})$ & 87 & $87 *$ \\
\hline Sodium fluoride (10mm) & 109 & $65 \cdot 2$ \\
\hline Oligomycin $(5 \mu \mathrm{g} . / \mathrm{ml})$. & 113 & $46 \cdot 8$ \\
\hline Quinolinate $(2.5 \mathrm{~mm})$ & 39 & 69 \\
\hline $\mathrm{CaCl}_{2}(2 \mathrm{~mm})$ & 75 & 8 \\
\hline GSH (10mm) & 129 & 102 \\
\hline GSSG (5 mM) & 104 & 78 \\
\hline Amytal (2.5 mM) & $97 \cdot 5$ & $16 \cdot 3 \dagger$ \\
\hline Mitochondria of rats & $123 \cdot 5$ & 86 \\
\hline
\end{tabular}

* $5 \mu \mathrm{M}$-triethyltin. $\dagger 2$ mm-Amytal.
The activity of the PEP carboxykinase in the supernatant was measured by the procedure of Seubert \& Huth (1965). The results showed that the amount of PEP produced by the mitochondria was $69 \cdot 2 \mu$ moles/g. dry wt./hr., whereas the amount produced by the solubilized PEP carboxykinase was only $26.5 \mu$ moles/g. dry wt./hr. Thus $38 \%$ of the formation of PEP in the mitochondria could be considered to be due to PEP carboxykinase. These calculations assume that all the soluble enzyme is extracted, that the mitochondrial enzymes are saturated with substrate and cofactors and that the kinetic properties of PEP carboxykinase are not affected by its extraction from the mitochondria and assay in dilute solution.

\section{DISCUSSION}

Production of PEP. In spite of the disrupted appearance of the mitochondria shown in the electron microscope (Plate 1) and the increase in permeability that is brought about by exposing mitochondria to solutions of low ionic strength it is still possible that portions of the mitochondria are not freely permeable to all the substances added in the Warburg vessel. Thus the experiments must be viewed with this limitation in mind. It must be conceded that if one of the substances added produces a definite response then that substance must have penetrated some part of the mitochondrion. A lack of response, on the other hand, could be due to a permeability barrier. Also, when calculations are made involving the concentration of solutes within the mitochondria, an even distribution of the solutes throughout the mitochondria has been assumed. This assumption may be incorrect.

With these provisos in mind the following suggest that the PEP formed in rat liver mitochondria does not involve PEP carboxykinase.

(1) Lack of involvement of high-energy nucleotides. The addition of ATP or GTP does not increase the amount of PEP formed from malate. Because of the high activity of nucleoside diphosphokinase in rat liver (Ishihara \& Kikuchi, 1968) it is to be expected that these two nucleotides would equilibrate. From all the numerous measurements of oxidative phosphorylation with rat liver mitochondria it is known that high-energy phosphate is incorporated more or less quantitatively into added nucleotides and that the product of substrate-level phosphorylation accompanying the oxidation of $\alpha$-oxoglutarate is recovered in a similar fashion. Equilibration of the nucleotides therefore occurs. Thus the drain on endogenous high-energy nucleotides imposed by dinitrophenol (either alone or with oligomycin) or hexokinase and glucose would be expected to decrease the amount of nucleotide available for PEP formation. Instead there is an 
increase in PEP formation. The experiments with labelled $P_{i}$ added in the presence of unlabelled GTP also strongly argue against the participation of GTP in the PEP formation. Zero equilibration of the added GTP with the endogenous pool would be necessary to explain the results with radioisotopes.

(2) Lack of influence of $\mathrm{Mg}^{2+}$. This should be compared with the stimulation of PEP formation in pigeon liver mitochondria where it is known that PEP carboxykinase is involved.

(3) Kinetic considerations. The $K_{m}$ values of PEP carboxykinase for GTP and oxaloacetate are given by Chang, Maruyama, Miller \& Lane (1966) as $1.6 \times 10^{-4} \mathrm{M}$ and $1.5 \times 10^{-4} \mathrm{M}$ respectively. Assuming that these two substrates are evenly distributed throughout the mitochondrial water and that the ratio (weight of mitochondrial water/mitochondrial dry weight) is 4 it can be calculated from the value given by Heldt \& Klingenberg (1965) that the concentration of total guanosine nucleotides could not exceed $60 \mu \mathrm{M}$. The water content of the mitochondria is usually greater than that assumed and it seems unlikely that all the guanosine nucleotides will be in the form of GTP.

The equilibrium for malate dehydrogenase is far in the direction of malate at $\mathrm{pH} \mathbf{7 \cdot 2}$ (equilibrium constant $2.33 \times 10^{-5}$; Stern, Ochoa \& Lynen, 1952). The measurements made by Birt \& Bartley (1960a) of the concentrations of $\mathrm{NAD}^{+}$and $\mathrm{NADH}$ in isolated rat liver mitochondria show that in a saline medium in the absence of substrate the ratio $\mathrm{NADH} / \mathrm{NAD}^{+} \simeq 0 \cdot 2$. With the concentration of malate used in the experiment this could result in a maximum concentration of oxaloacetate of $0.5 \mu \mathrm{M}$. Further, Birt \& Bartley (1960b) showed that the ratio $\mathrm{NADH} / \mathrm{NAD}^{+}$became 3 in the presence of malate and $0.1 \mathrm{~mm}$-dinitrophenol. This would decrease the equilibrium concentration of oxaloacetate even further. It is difficult to believe that PEP carboxykinase can produce PEP under these conditions. It is also significant that the yield of PEP did not increase appreciably above an initial concentration of $1 \mathrm{~mm}$-malate. This suggests that the reaction is not dependent on the concentration of oxaloacetate. Such questions about kinetic requirements of PEP carboxykinase have been raised by Greville \& Tubbs (1968).

(4) Comparison of the effects of substances on PEP carboxykinase with the effects of the same substances on mitochondrial PEP formation. These experiments must be viewed with the following provisos in mind.

(a) The PEP carboxykinase may show different kinetic properties when solubilized and diluted from those exhibited within the mitochondria.

(b) We have assumed that extraction of freezedried mitochondria with water gives a virtually quantitative extraction of PEP carboxykinase. As shown by Ballard \& Hanson (1967) this gives the maximum PEP carboxykinase activity compared with other procedures.

The maximum activity of PEP carboxykinase extracted from the mitochondria under conditions of substrate saturation was much less in terms of PEP production than the amount of PEP produced in the mitochondrial preparation without added cofactors and at low substrate concentrations. In no case is there any parallelism between the effect of inhibitors on PEP carboxykinase activity and the amount of PEP produced in the mitochondrial system.

Further, Stickland (1959) showed that PEP carboxykinase was inhibited by potassium chloride above $10 \mathrm{~mm}(90 \%$ at $0 \cdot 1 \mathrm{M})$. In our experiments potassium chloride was always above $40 \mathrm{~mm}$ and an increased concentration had no effect on PEP production. Also, unpublished work (B. Dean \& W. Bartley) has shown that in mitochondria washed with water PEP production is dependent on the presence of potassium chloride and increases with increase in potassium chloride concentration.

The evidence therefore strongly suggests that rat liver mitochondrial PEP cannot be formed through the PEP carboxykinase route.

On the other hand if it is assumed that the PEP formed by the rat liver mitochondria is formed through the PEP carboxykinase reaction, that reaction must in some way be segregated from the rest of the metabolic pool. This follows from a simple consideration of the kinetic characteristics of the enzyme since the overall concentrations of oxaloacetate that result from malate dehydrogenase are much too low to result in appreciable PEP synthesis and the PEP formation is independent of added GTP. Such segregation is possible but cannot be defined anatomically since PEP carboxykinase is a soluble enzyme. Such segregation also poses the problem that the segregated portion of the mitochondria must be at the same time accessible to the substrates of the reaction. For example the oxaloacetate formed by the malate dehydrogenase (a soluble enzyme) must move to the site of action of the PEP carboxykinase and there be concentrated in some way to overcome the unfavourable kinetics. Similarly the GTP formed in the $\alpha$-oxoglutarate oxidase system must be immediately accessible to PEP carboxykinase without equilibrating with added GTP or being subject to nucleoside diphosphate kinase. Similar incongruities appear in other cases where the effect of the inhibitors tested require some form of segregation of PEP carboxykinase for this to be the route of PEP production. Any segregation theory requires that there be at least two pools of PEP carboxykinase, one accessible to and inhibited by quinolinate and the other not. 
Because of these and other similar arguments it appears simpler to postulate a route of PEP formation not involving PEP carboxykinase in rat liver mitochondria.

Production of pyruvate. Production of pyruvate presumably arises either by the spontaneous decarboxylation of oxaloacetate, by the breakdown of an intermediate common to the production of PEP and pyruvate from malate, or from PEP itself. The pyruvate kinase activity of the mitochondria measured in the presence of saturating amounts of fructose 1,6-diphosphate is $150 \mu$ moles/g. dry wt. of mitochondria/hr. At the low concentrations of fructose diphosphate and PEP present in the mitochondria under the experimental conditions used, the activity of the pyruvate kinase is likely to be much less than (possibly one-tenth) that measured in the presence of the saturating concentrations of fructose diphosphate and PEP. However, it is conceivable that pyruvate could arise from PEP. In this case the sum of PEP and pyruvate would be the minimum amount of PEP formed and, since the oxygen uptake normally exceeds the PEP formation by about tenfold, much of the pyruvate could represent the steady concentration of the substance, in which its production by pyruvate kinase is balanced by further oxidation. The accumulation of pyruvate in the presence of arsenite would then reflect the inability of pyruvate to be oxidized because of the blocking of pyruvate oxidase. The lowered accumulation of pyruvate in the presence of $0.01 \mathrm{~mm}$-dinitrophenol is brought about by the extra rate of oxidation of the pyruvate, as shown by the increased rate of respiration that occurs with this concentration of dinitrophenol. Such an explanation does not account for the diminution in PEP formation in the presence of arsenite, unless arsenite has a second point of action in the reaction sequence; nor can it account for the diminution in the amount of pyruvate which accumulates at higher concentrations of dinitrophenol when respiration is decreased. Any such explanations would require dinitrophenol to have an inhibitory effect on pyruvate kinase.

The evidence is therefore substantially against pyruvate kinase as the source of pyruvate accumulation.

The possibility of 'malic' enzyme as a source of pyruvate is not supported by the measurement of the activity of this enzyme in the mitochondrial preparations. At most $50 \mu$ moles of pyruvate could be formed/g. dry wt./hr. and over $90 \mu$ moles of pyruvate are produced in the presence of arsenite. Also, any involvement of 'malic' enzyme requires an inhibition by dinitrophenol in all circumstances when respiration does not increase and this does not occur. Thus the evidence is also against 'malic' enzyme as the source of pyruvate.
Pyruvate can arise by the spontaneous decarboxy. lation of oxaloacetate but there seems to be no estimate for the rate of this reaction and there seems no reason to suppose that dinitrophenol influences this rate of decarboxylation. Because of the high activity of malate dehydrogenase and the unfavourable equilibrium of the malate dehydrogenase reaction for the formation of oxaloacetate it seems unlikely that the low concentration of oxaloacetate (about $0.5 \mu \mathrm{M}$ ) in the mitochondria could produce an appreciable rate of pyruvate formation by spontaneous decarboxylation.

\section{REFERENCES}

Alberti, K. G. M. M. \& Bartley, W. (1969). Biochem.J. 111, 763.

Amoore, J. E. \& Bartley, W. (1958). Biochem. J. 69, 223. Ballard, F. J. \& Hanson, R. W. (1967). Biochem. J. 104, 866.

Ballard, F. J. \& Oliver, I. T. (1963). Biochim. biophys. Acta, 71, 578.

Bartley, W. (1955). Biochem.J. 61, 555.

Bartley, W. (1961). Biochem. J. 80, 46.

Bartley, W. \& Amoore, J. E. (1958). Biochem. J. 69, 348.

Bartley, W. \& Avi-Dor, Y. (1955). Biochem. J. 59, 194. Bartley, W. \& Enser, M. B. (1964). Biochem. J. 98, 322. Birt, L. M. \& Bartley, W. (1960a). Biochem. J. 75, 303. Birt, L. M. \& Bartley, W. (1960b). Biochem. J. 76, 427.

Chang, H. C., Maruyama, H., Miller, R. S. \& Lane, M. D. (1966). J. biol. Chem. 241, 2421.

Czok, R. \& Eckert, L. (1963). In Methods of Enzymatic Analysis, p. 229. Ed. by Bergmeyer, H. U. New York and London: Academic Press.

Davis, E. J. \& Gibson, D. M. (1967). Biochem. biophys. Res. Commun. 29, 815.

Gamble, J. L., jun. \& Mazur, J. A. (1967). J. biol. Chem. $242,67$.

Gevers, W. (1967). Biochem. J. 103, 141.

Greville, G. D. \& Tubbs, P. K. (1968). In Essays in Biochemistry, vol. 4, p. 155. Ed. by Campbell, P. N. \& Greville, G. D. London and New York: Academic Press.

Hanes, C. S. \& Isherwood, F. A. (1949). Nature, Lond., 164, 1107.

Heldt, H. W. \& Klingenberg, M. (1965). Biochem. Z. 343, 433.

Ishihara, N. \& Kikuchi, G. (1968). Biochim. biophys. Acta, 153, 733.

Lamprecht, W. \& Trautschold, I. (1963). In Methods of Enzymatic Analysis, p. 543. Ed. by Bergmeyer, H. U. New York and London: Academic Press.

Lehninger, A. L. (1962). Physiol. Rev. 42, 467.

Mudge, G. H., Neuberg, H. W. \& Stanbury, S. W. (1954). J. biol. Chem. $210,965$.

Sanadi, D. R., Langley, M. \& White, F. (1959). J. biol. Chem. 234, 183.

Seubert, W. \& Huth, W. (1965). Biochem. Z. 343, 176.

Shrago, E., Lardy, H. A., Nordlie, R. C. \& Foster, D. 0. (1963). J. biol. Chem. 238, 3188.

Shrago, E., Young, J. W. \& Lardy, H. A. (1964). Biochemistry, 3, 1687. 
Stadtman, E. R. (1957). In Methods in Enzymology, vol. 3, p. 931. Ed. by Colowick, S. P. \& Kaplan, N. O. New York: Academic Press Inc.

Stanbury, S. W. \& Mudge, G. H. (1954). J. biol. Chem. 210, 949.

Stern, J., Ochoa, S. \& Lynen, F. (1952). J. biol. Chem. 198, 313.
Stickland, R. G. (1959). Biochem. J. 73, 660.

Utter, M. F. \& Kurahashi, K. (1954). J. biol. Chem. 207, 821.

Veneziale, C. M., Walter, P., Kneer, N. \& Lardy, H. A. (1967). Biochemistry, 6, 2129.

Werkheiser, W. C. \& Bartley, W. (1957). Biochem. J. 66, 79.

Yeung, D. \& Oliver, I. T. (1967). Biochem. J. 105, 1229. 